\title{
Topical issue on underground nuclear astrophysics and solar neutrinos: Impact on astrophysics, solar and neutrino physics
}

Published online: 19 April 2016 - (c) Società Italiana di Fisica / Springer-Verlag 2016

In 1958 the measurement of the ${ }^{3} \mathrm{He}(\alpha, \gamma){ }^{7}$ Be cross section by Holmgren and Johnston gave a value a thousand times higher than expected. This result showed that the dream of detecting solar neutrinos to study the deep interior of the Sun could become reality using a chlorine target. As we see, nuclear astrophysics and solar neutrinos have been tightly bound since the beginning of the solar neutrino saga. Such bond became stronger and stronger over the years, when the measurement of a solar neutrino flux lower than expected triggered a great deal of hypotheses on the possible reasons: from unknown resonances in the cross sections to new neutrino properties, as oscillations or magnetic moment.

The Editorial Board of The European Physical Journal A (EPJA) felt it is now time to have a review on the main results and on the future perspectives for the two correlated fields of underground nuclear astrophysics and solar neutrinos.

Underground nuclear astrophysics was born twenty five years ago in the core of Gran Sasso, when LUNA (Laboratory for Underground Nuclear Astrophysics) started its activity with a $50 \mathrm{kV}$ accelerator in the same laboratory where two milestone experiments for solar neutrino detection, Gallex/GNO and Borexino, were also active. LUNA still remains the only experiment in the world running an accelerator deep underground (at the moment a $400 \mathrm{kV}$ accelerator with hydrogen and helium beams). Its achievements have triggered two similar facilities which are now going to start in the Republic of China and in the United States.

As a matter of fact, the extremely low laboratory background has allowed for the first time nuclear physics experiments with very small count rates, down to a couple of events per month. This way, the important reactions responsible for the hydrogen burning in the Sun could be studied down to the relevant stellar energies. In particular, the clear exclusion of the "ghost" resonance in the cross section of ${ }^{3} \mathrm{He}\left({ }^{3} \mathrm{He}, 2 \mathrm{p}\right){ }^{4} \mathrm{He}$ within the solar Gamow peak and the precise measurement of ${ }^{3} \mathrm{He}(\alpha, \gamma){ }^{7}$ Be have firmly established the correctness of the nuclear ingredients of the proton-proton chain in the Standard Solar Model.

Equally important, the direct measurement of the bottle-neck reaction of the CNO cycle, ${ }^{14} \mathrm{~N}(\mathrm{p}, \gamma){ }^{15} \mathrm{O}$, at very low energy has provided a cross section lower by about a factor of two than the existing extrapolations decreasing, by the same amount, the flux of CNO neutrinos from the Sun and increasing, by about one billion years, the limit on the age of the Universe. Furthermore, the LUNA results paved the way to the study of the metallicity in the Sun core through the forthcoming measurement of the CNO solar neutrinos.

In addition to the solar phase of LUNA, the rich program lasting for several years and devoted to the study of the Big Bang nucleosynthesis and of the nucleosynthesis of the elements through the $\mathrm{CNO}, \mathrm{Mg}-\mathrm{Al}$ and $\mathrm{Ne}-\mathrm{Na}$ cycles in different stars is also extensively reviewed. The motivation here is to reproduce the abundance of the light elements and to identify the production site in stellar scenarios different from the Sun: hydrogen burning at the higher energies corresponding to the hydrogen shell of Asymptotic Giant Branch (AGB) stars or to the explosive phase of classical Novae.

Finally, the next two steps beyond hydrogen burning are discussed: helium and carbon burning. Their study will be performed with the new 3.5 single-ended accelerator which is going to be installed in Gran Sasso at the beginning of 2018. The accelerator will provide hydrogen, helium and carbon (also double-ionized) beams and it will be devoted to the study of those key reactions of helium and carbon burning which are determining and shaping both the evolution of massive stars towards their final fate and the nucleosynthesis of most of the elements in the nuclide chart. 
For the sake of completeness, there is also an exhaustive review of the Trojan Horse method, an indirect method which is able to provide cross section measurements free from the electron screening effect down to extremely low energies, completely covering the region of astrophysical interest.

The second part of this issue is devoted to neutrinos. As a matter of fact, the phenomenon of the neutrino oscillation has been and is still, for some aspects, one of the most important breakthroughs in particle physics in the last decades. This is evidenced also by the 2015 Nobel Prize in Physics, assigned to experiments on the neutrino oscillation. The study of the oscillations has been triggered by the so-called "solar neutrino problem", and the solar neutrinos allowed to achieve milestones in our understanding of this phenomenon.

The great advantage of the Sun as a neutrino source is the very low energy of the neutrinos and the large distance between production and detection sites. The transition probability between neutrino flavors is strongly enhanced by these experimental conditions and the solar neutrinos are an ideal choice for these researches.

The values of the free oscillation parameters - the mass difference between the eigenstates of the neutrino masses and the mixing angle - are now measured with high precision, while the MSW model can be considered experimentally confirmed, at least for what concerns the vacuum and matter regimes, thanks to SNO, Superkamiokande and Borexino experiments. The Cherenkov experiments explored the high-energy part of the solar neutrino energy spectrum (namely the high-energy tail of the neutrinos produced by the ${ }^{8} \mathrm{~B}$ reaction), while Borexino, due to its unprecedented radiopurity, has been and is able to study the entire spectrum over $\sim 200 \mathrm{keV}$, and then to detect the oscillation in vacuum and to measure the ratio between the electron neutrino survival probabilities in vacuum and in matter.

Some problems are still open, like the study of the transition zone between the oscillations in vacuum and in matter, a zone which is strongly influenced by the currently much debated hypothesis of the existence of the Non-Standard neutrino Interaction. Borexino measured the neutrino rate from pep reaction, which is falling just in the transition region, but the uncertainty of this experimental rate is still too large. In addition, the hierarchy of neutrino masses is not yet measured, as the study of the oscillations gives only the mass differences.

The neutrino oscillation is a long saga, started with the pioneering radiochemical experiments, and continued with the Cerenkov experiments, like SNO and SuperKamiokande, and the experiments with liquid scintillator, like BOREXINO and Kamland (the latter was focused mainly on reactor antineutrinos). Experiments with accelerator beams, like LSND and MiniBooNE, showed results not easy to be interpreted and, in some respects, even contradictory. But the hypothesis of a third mass difference is not completely ruled out, although unlikely; it would be added to the mass differences related to the solar and atmospheric neutrinos. A third mass difference needs a fourth neutrino, the sterile neutrino, which currently many experiments, either in design or in construction phase, plan to study. Borexino is planning an ad hoc measurement using an artificial source.

The study of solar neutrinos has a major impact not only on neutrino physics, but also on solar physics. Especially the results of BOREXINO, which measured all solar neutrino fluxes of the major solar cycle (pp), confirmed that the nuclear fusion reactions are the source of the solar energy, as foreseen by the Standard Solar Model; Borexino provided also a measurement of the solar luminosity through neutrinos, getting good agreement with the same luminosity measured via the photons emitted by our Star (that need about 100000 years to escape from the Sun). What is not yet fixed is the high-low metallicity puzzle, which could be settled via a good measurement of the CNO neutrinos.

Our knowledge of the transition region could be improved by the future new results of Borexino, which is making an effort to measure the pep flux, the ${ }^{8} \mathrm{~B}$ flux in the energy range $3-5 \mathrm{MeV}$, and the CNO flux with higher precision. Also Superkamiokande is trying to measure the ${ }^{8} \mathrm{~B}$ flux pushing down the threshold to $2.5-3 \mathrm{MeV}$. All these measurements fall just in the transition region.

The determination of the neutrino mass hierarchy is one of the main goals of the Chinese experiment Juno, exposed to reactor antineutrinos, which is planned to start the data taking in 2020. A further experiment that could give an important contribution to the study of the solar neutrinos is $\mathrm{SNO}+$, which has a volume 30 times larger than Borexino, but a question mark concerns the level of radio-purity which the experiment will be able to reach.

In this Topical Issue the experimental methods of the neutrino detectors, the experiments, the analysis approaches which follow the various detection techniques, the physics results, and their impact on the neutrino and the solar physics, are described and deeply discussed.

Gianpaolo Bellini, Carlo Broggini, and Alessandra Guglielmetti 